\title{
EFECTOS DE UN PROGRAMA DE HABILIDADES SOCIALES CONSIDERANDO LA INTELIGENCIA EMOCIONAL ${ }^{1}$ Effects of a social skills program considering emotional intelligence
}

\author{
Luciana del Rosario Távara Castillo* \\ María del Rosario Elizabeth Torres Oporto**
}

\begin{abstract}
Resumen
La investigación fue realizada con el propósito de demostrar que la aplicación de un programa de refuerzo de las habilidades sociales en niños de una institución educativa incrementa dichas habilidades, considerando los componentes de la inteligencia emocional de BarOn. La muestra fue no probabilística intencional, conformada por 17 alumnos de 8 a 10 años de edad de 4to grado de primaria, de ambos sexos; el diseño de investigación correspondió a diseños preexperimentales, diseño pre y postest con un solo grupo. Se aplicó el Inventario de Inteligencia Emocional de BarOn ICE: NA; y el Programa de Habilidades Sociales de Vallés Arándiga, adaptado por las autoras de la investigación. Para el análisis estadístico se utilizó la prueba no paramétrica de Wilcoxon, demostrándose que la aplicación del programa de habilidades sociales mejoró significativamente dichas habilidades, considerando los componentes de la inteligencia emocional de BarOn.
\end{abstract}

Palabras clave: Habilidades sociales, inteligencia emocional

\begin{abstract}
The research was conducted to demonstrate that the implementation of a program to improve children's social skills at an educational institution, does increase such skills, considering the components of the Bar-On model of emotional-social intelligence. The non-probabilistic purposive sample consisted of 17 students from 8-10 years old of 4th grade elementary school, of both sexes; a pre experimental research with pretest and posttest designs with just one group were used. The Questionnaire Inventory Ratio ICE-BarOn Emotional Intelligence and the Social Skills Program of Arándiga Vallés, adapted by the authors of the research were used. For statistical analysis, nonparametric Wilcoxon test was used, showing that the implementation of the program of social skills improved significantly such skills, considering the components of emotional intelligence of BarOn.
\end{abstract}

Keywords: Social skills, emotional intelligence

1 El presente artículo está basado en la tesis de maestría en Psicología de las autoras, titulada Efectos de un programa de refuerzo de las habilidades sociales en niños considerando la inteligencia emocional, sustentada en el año 2012 en la Universidad Femenina del Sagrado Corazón. Lima, Perú.

* Magíster en Psicología con mención en Psicología Educativa y Problemas de Aprendizaje de la Universidad Femenina del Sagrado Corazón. lucianatavaracastillo@hotmail.com

** Magíster en Psicología con mención en Psicología Educativa y Problemas de Aprendizaje de la Universidad Femenina del Sagrado Corazón.elita1907@gmail.com 
El ser humano es un ser gregario por excelencia que a lo largo de su vida está sometido a procesos de socialización, adquiriendo hábitos que le permiten relacionarse adecuadamente con su entorno familiar y social.

Las habilidades sociales son inherentes al ser humano pero requieren de acciones específicas para su desarrollo y perfeccionamiento. La calidad de las habilidades sociales depende, en gran medida, de las acciones de capacitación y educación a las que han sido expuestos los alumnos. El cultivo y desarrollo de estas habilidades favorecerá a la capacidad de la persona de permanecer socialmente incluida en todos los ámbitos de la vida: hogar, escuela, trabajo, grupo de amigos, comunidad.

En la escuela, la modalidad que favorece el desarrollo de las habilidades sociales es la relación mutua entre docente-alumno, y alumno-alumno, así como también, los procesos didácticos formulados y construidos en el ámbito escolar.

Las habilidades sociales han sido objeto de una marcada atención en los últimos años, debido fundamentalmente a la constatación de su importancia en la infancia y adolescencia. La falta de habilidad social está relacionada a varias dificultades: baja aceptación, rechazo, aislamiento, problemas escolares, desajustes psicológicos, delincuencia juvenil, etc. Es necesario, por ende, promover la enseñanza de la conducta interpersonal en la población de niños y jóvenes, con el objeto de incrementar su competencia social, prevenir posibles desajustes de conductas y, en definitiva, contribuir al desarrollo integral.

Al desarrollar un programa de refuerzo de las habilidades sociales considerando la inteligencia emocional, estamos pretendiendo que el estudiante aprenda a respetarse como persona, aprenda a convivir, aprenda a pensar y resolver los problemas que se le presentan y afrontar adecuadamente sus relaciones sociales, logrando tener un mayor manejo de sus virtudes, emociones y control de sus impulsos. Es decir, logrará ejercer dominio sobre sus propias emociones y responderá apropiadamente a diversas situaciones de la vida.

La inteligencia emocional -según Goleman (1998)- permite conseguir el éxito personal (en aspectos como la felicidad, comunicación eficaz, solución de conflictos, adaptación a las circunstancias, entre otros) haciendo uso no solo del manejo de nuestra emocionalidad, sino también de nuestros conocimientos y habilidades empáticas.

Siguiendo con Goleman (1998) -y citando en extenso- las habilidades emocionales pueden ser definidas como

Capacidades y disposiciones para crear voluntariamente un estado de ánimo o sentimiento a partir de las ideas que tenemos sobre lo que ocurre. De ahí que es necesario aprender a atribuir significados emocionalmente deseables a los acontecimientos que tienen lugar en las relaciones que establecemos con los demás. De este modo, conociendo qué pensamiento y comportamientos provocan nuestros estados de ánimo, podemos manejarlos mejor para solucionar los problemas que aquellos generan. La inteligencia es precisamente eso, la capacidad de solucionar problemas adaptándose a las circunstancias. Cuando los problemas son de índole emocional, son las habilidades emocionales las que se ponen en práctica para alcanzar mayores niveles de satisfacción y de desarrollo personal. Para que ello se produzca, no debe olvidarse la dimensión social que enmarca la expresión emocional de nuestros estados de ánimo. Es decir que las habilidades sociales deben vincularse con las emocionales, de tal modo que las tres dimensiones del comportamiento: pensar, sentir y hacer (respuestas cognitivas, afectivas y conductuales) queden integradas. (p. 91)

\section{MÉTODO}

La presente investigación es de tipo tecnológica de nivel experimental según Sánchez y Reyes (1986). El diseño, según Alarcón (1991), corresponde al grupo de diseños experimentales; específicamente se ubica dentro de los preexperimentales, teniendo el nombre de diseño pretest y postest con un solo grupo.

\section{Participantes}

Los participantes de este estudio fueron 17 alumnos de 8 a 10 años de edad, del 4to grado A del nivel de primaria de una institución educativa personalizada. 
La muestra es no probabilística intencional porque ha sido elegida para satisfacer los requerimientos de las variables de estudio.

\section{Instrumento}

Se utilizó el Programa de Refuerzo de Habilidades Sociales de Vallés Arándiga (Vallés, 2005) y el Inventario de Inteligencia Emocional de BarOn ICE: NA (BarOn, 1997) en Niños y Adolescentes, adaptado por Nelly Ugarriza Chávez y Liz Pajares del Águila (2006).

\section{Procedimiento}

Se planteó el problema a estudiar y se identificaron las variables de estudio. Se establecieron los objetivos, se realizaron los estudios previos relacionados con esta investigación y se determinó la población y muestra de estudio. Se concretó el tipo y diseño de la investigación. Se revisó el Inventario de Inteligencia Emocional de BarOn ICE: NA y el Programa de Refuerzo de las Habilidades Sociales, modificándose las palabras españolas a palabras peruanas, para lo cual se solicitó el criterio de cuatro jueces para avalar la adaptación del programa. Se elaboró el esquema y la redacción de los temas que forman parte del marco teórico. De la misma manera, se procedió a la aplicación del Inventario de Inteligencia Emocional de BarOn ICE: NA, antes y después del Programa, el cual tuvo una duración de cinco meses. Posteriormente se procedió al análisis estadístico utilizando la prueba no paramétrica de Wilcoxon, llegando así a la presentación, interpretación y discusión de los resultados. Finalmente, se realizaron las conclusiones $\mathrm{y}$ recomendaciones.

\section{RESULTADOS}

Para comparar los efectos del programa de refuerzo de las habilidades sociales considerando la inteligencia emocional, se ha utilizado la prueba no paramétrica de Wilcoxon, pues se trata de comparar dos mediciones en los mismos participantes. Los resultados de estas comparaciones fueron:

- En relación a la hipótesis general, la aplicación del programa incrementó significativamente el nivel de las habilidades sociales en niños de 8 a 10 años, expresados en los componentes emocionales de BarOn, encontrando que en la comparación de los puntajes obtenidos en la inteligencia emocional referido al componente emocional total, existe un rango promedio de 9 para lo positivo, y un puntaje $\mathrm{z}$ igual a 3.651 , significativo al nivel del 0.05 , por lo cual puede señalarse que sí existen diferencias significativas entre ambas mediciones; dicho componente emocional está relacionado con el área de habilidades sociales del programa.

- Así mismo al verificar las hipótesis, sobre la existencia de diferencias significativas en los componentesemocionalesintrapersonal,interpersonal, manejo del estrés, adaptabilidad, estado de ánimo general,impresión positiva, y el componente emocional total (hipótesis 1, 2, 3, 4, 5, 6 y 7, respectivamente), antes y después de la aplicación del programa, se encontró en la comparación de los puntajes obtenidos lo siguiente:

. Intrapersonal: Hay un rango promedio de 9 para lo positivo, y un puntaje z igual a -3.642 , significativo al nivel del 0.05 , por lo cual puede señalarse que sí existen diferencias significativas entre ambas mediciones; dicho componente está relacionado con el área de autoestima del programa.

- Interpersonal: Hay un rango promedio de 9 para lo positivo, y un puntaje $\mathrm{z}$ igual a -3.642 , significativo al nivel del 0.05 , indicando la existencia de diferencias significativas entre ambas mediciones; este componente está relacionado con el área de habilidades sociales del programa.

- Manejo del estrés: Hay un rango promedio de 9 para lo positivo, y un puntaje z igual a -3.625 , significativo al nivel del 0.05 , por lo cual puede señalarse que sí existen diferencias significativas entre ambas mediciones, dicho componente está relacionado con el área de solución de problemas del programa.

. Adaptabilidad: Hay un rango promedio de 9 para lo positivo, y un puntaje z igual a -3.637 , significativo al nivel del 0.05 , señalando así la existencia diferencias significativas entre ambas mediciones; este componente está relacionado con el área de solución de problemas del programa.

.Estado de ánimo general: Hay un rango promedio de 8.5 para lo positivo, y un puntaje z igual a -3.589 , significativo al nivel del 0.05 , indicando la existencia 
de diferencias significativas entre ambas mediciones; dicho componente está relacionado con el área de autoestima del programa.

Impresión positiva: Hay un rango promedio de 9 para lo positivo, y un puntaje z igual a -3.684 , significativo al nivel del 0.05 , por lo cual puede señalarse que sí existen diferencias significativas entre ambas mediciones; este componente está relacionado con el área de autoestima del programa.

. Para finalizar, en el componente emocional total hay un rango promedio de 9 para lo positivo, y un puntaje z igual a -3.651 , significativo al nivel del 0.05 , señalando así la existencia de diferencias significativas entre ambas mediciones. Dicho componente emocional está relacionado con el área de habilidades sociales del programa de refuerzo de las habilidades sociales (véase la tabla 1).

Al realizar la comparación de los puntajes de los componentes de inteligencia emocional antes y después de la aplicación de un programa de refuerzo de las habilidades sociales, se verifica la hipótesis general, es decir la aplicación de un Programa de Refuerzo de las Habilidades Sociales incrementa significativamente el nivel de las habilidades sociales en niños de 8 a 10 años expresados en los componentes emocionales de BarOn,

Tabla 1

Puntaje del componente de inteligencia emocional total relacionado con el área de habilidades sociales antes y después de la aplicación de un programa de refuerzo de las habilidades sociales

\begin{tabular}{lcccc}
\hline & $\begin{array}{c}\text { Rango } \\
\text { promedio }\end{array}$ & $\begin{array}{c}\text { Suma } \\
\text { de rangos }\end{array}$ & $\mathrm{Z}$ & $\mathrm{N}$ \\
\hline Rangos negativos & 0 & 0 & $-3.651^{*}$ & 17 \\
Rangos positivos & 9.0 & 153.00 & & \\
Empates & - & & & \\
\hline
\end{tabular}

$* \mathrm{p}<0.05$

\section{DISCUSIÓN}

Los resultados permitieron comprobar la hipótesis general formulada, evidenciando de esta manera la efectividad del Programa de Refuerzo de las Habilidades Sociales considerando la Inteligencia Emocional, el cual produjo una mejora significativa en los alumnos, ya que se observó que empezaron a interactuar adecuadamente, expresaban sus sentimientos y emociones asertivamente, comenzaron a ser responsables, respetaban el turno de la palabra, siendo tolerantes entre ellos.

Porotroladoencontramosdiferenciassignificativas en los componentes emocionales: intrapersonal, interpersonal, manejo del estrés, adaptabilidad, estado de ánimo general, impresión positiva, y el componente emocional total, antes y después de la aplicación del programa, tal y como se planteó en las hipótesis 1,2, $3,4,5,6$ y 7 , respectivamente. En relación a nuestros resultados observamos que el componente:

a) Intrapersonal, está relacionado con el área de autoestima de dicho programa, acorde con lo que plantea Coopersmith (1981):

La autoestima es la evaluación que una persona realiza y que generalmente mantiene sobre sí, esta valoración se expresa a través de sus actitudes y que constituye finalmente una expresión de aprobación o desaprobación, indicando el grado de que cada persona se considera competente y exitosa. (p. 5).

Esto se corrobora con lo que plantea BarOn (1997) en el componente intrapersonal, cuando define su subcomponente de autoconcepto como "la habilidad para comprender, aceptar y respetarse a sí mismo, aceptando nuestros aspectos positivos y negativos así como nuestras limitaciones y posibilidades" (p.14).

b) Interpersonal, se relaciona con el área de habilidades sociales del programa mencionado, acorde con lo que plantea Gil (1993) quien ofrece una amplia y aclaratoria definición, síntesis de otras definiciones dadas por diversos autores:

Las habilidades sociales son conductas que se manifiestan en situaciones interpersonales, estas conductas son aprendidas, y por tanto pueden 
ser enseñadas. Estas conductas se orientan a la obtención de distintos tipos de reforzamiento, tanto del ambiente (consecuencia de objetos materiales o refuerzos sociales) como auto refuerzos. (p. 270)

Asimismo, ello se confirma con lo planteado por BarOn (1997) en el componente interpersonal, cuando define su subcomponente de relaciones interpersonales como "la habilidad para establecer y mantener relaciones mutuas satisfactorias que son caracterizadas por una cercanía emocional e intimidad" (p.14). Igualmente, Pelechano (1984) define las habilidades interpersonales como:

Un patrón complejo de respuestas que llevan a una confianza y a un reconocimiento personal por parte de los demás y resultan eficaces para ejercer un autocontrol así como una influencia (directa o indirecta) sobre los demás, con la utilización de medios y procedimientos socialmente admisibles. (p. 393).

c) Manejo del estrés, está relacionado con el área de solución de problemas, acorde con lo que se plantea en el programa clima de clases, el cual enfatiza Vallés (1997) en su programa de solución de conflictos interpersonales III:

Desde un enfoque cognitivo y eminentemente preventivo prepara a los alumnos y alumnas para afrontar con eficacia las situaciones de interacción con los iguales y los adultos que resultan problemáticas para la adecuada convivencia escolar. Para el entrenamiento de solución de problemas interpersonales deben tenerse en consideración los siguientes elementos: La identificación del problema, la generación de soluciones, la previsión de las consecuencias y la aplicación de la solución elegida. (p. 3).

Lo cual es apoyado por BarOn (1997) en el componente manejo del estrés cuando define su subcomponente de tolerancia al estrés como "la habilidad para soportar eventos adversos, situaciones estresantes y fuertes emociones sin desmoronarse, enfrentándose activa y positivamente al estrés" (p.14). d) Adaptabilidad, está relacionado con el área de solución de problemas, de acuerdo con lo que planteara Antonio Vallés (2005) y corroborado por BarOn en el componente adaptabilidad cuando define su subcomponente de solución de problemas como "la habilidad para identificarse y definir los problemas, generar e implementar soluciones afectivas" (1997, p.14).

e) Estado de ánimo en general, está vinculado con el área de autoestima, acorde con lo que afirmara Antonio Vallés (1997) "la autoestima es: sentirse a gusto consigo mismo, sentirse importante, estar feliz con las cosas adecuadas que haces, aceptar las dificultades e intentar solucionarlas, no desanimarse cuando las cosas no van bien" (p. 20). Lo cual se confirma con lo que planteara BarOn (1997) en el componente estado de ánimo cuando define su subcomponente de felicidad como "la habilidad para sentirse satisfecho con nuestra vida, para disfrutar de sî" (p.14), y el subcomponente optimismo como "la habilidad para ver el aspecto más brillante de la vida y mantener una actitud positiva a pesar de la adversidad y los sentimientos negativos" (p.15).

f) Impresión positiva, está relacionado con el área de autoestima, acorde con lo planteado por Abraham Maslow (1954) quien considera que:

La autoestima es la base y centro del desarrollo humano; la autoestima es conocimiento, concientización, y práctica de todo el potencial de cada individuo. Solo se podrá respetar a los demás cuando uno se respete a sí mismo: solo podemos dar cuando nos hemos dado a nosotros mismos, solo podremos amar cuando nos amemos a nosotros mismos. (p. 3)

Asimismo BarOn (1997) lo reafirma en el componente intrapersonal cuando define su subcomponente de autorrealización como "la habilidad para realizar lo que realmente podemos, queremos y disfrutamos de hacerlo" (p. 14).

g) Componente emocional total, está vinculado con el área de habilidades sociales, acorde con lo que plantea Antonio Vallés (2005): "las habilidades sociales como las conductas que debemos tener cuando nos relacionamos con los demás para llevarnos 
bien con todos y no tener problemas: es la forma en que decimos las cosas" (p. 7). Igualmente BarOn lo plantea en el componente interpersonal cuando define su subcomponente de empatía como "la habilidad para percatarse, comprender y apreciar los sentimientos de los demás" (p. 14), y su subcomponente de responsabilidad social como "la habilidad para demostrarse a sí mismo como una persona que coopera, contribuye y que es un miembro constructivo del grupo social" (p. 14).

\section{CONCLUSIONES}

La aplicación del programa de refuerzo de las habilidades sociales incrementa significativamente el nivel de las habilidades sociales expresadas en los componentes emocionales de BarOn (1997), en donde se observa que los alumnos progresan en la forma de enfrentar las demandas de su vida cotidiana.

Asimismo se aprecia una mejoría en cada uno de los componentes, así en el intrapersonal: comprendieron sus sentimientos y emociones, y el por qué de los mismos; en el interpersonal: mejoraron su forma de interactuar y de saber conversar de manera adecuada con los demás, evidenciándose en sus cambios verbales, ya que empezaron a utilizar palabras como "gracias", "por favor", "permiso", así como mostrar conductas de cortesía y amabilidad; en el manejo del estrés: enfrentaron situaciones de tensión, en donde aprendieron a trabajar bajo presión; en el componente adaptabilidad: se adaptaron a las exigencias del entorno, enfrentando de manera positiva las situaciones problemáticas que se les presentaban lo cual contribuyó a disminuir significativamente la distractibilidad cuyos cambios producidos favorecieron a que logren completar sus tareas y por ende un mejor rendimiento académico. En el estado de ánimo en general: aprendieron a tener una apreciación positiva y optimista sobre las diferentes situaciones que se les presentaba en su quehacer diario; en impresión positiva: tuvieron una mejor apreciación de sí mismos; por último, en el componente emocional total: enfrentaban de manera adecuada las demandas de la vida diaria.

Es importante desarrollar habilidades sociales en los alumnos(as), padres de familia, educadores, autoridades y sociedad en general, ya que, aprendiendo a cultivarlas se puede construir una comunidad tolerante, participativa y democrática, establecer metas comunes y proponer caminos apropiados para conseguirlas, ejecutar planes y evaluar sus resultados con fines de perfeccionamiento.

\section{REFERENCIAS}

Alarcón, R.(1991). Métodos y diseños de investigación del comportamiento. Lima: Universidad Peruana Cayetano Heredia. Fondo Editorial.

BarOn, R. (1997). BarOn Emotional Quotient Inventory (EQ-i): Technical manual. Toronto, Canada: MultiHead Systems Inc.

Coopersmith, S. (1981). The antecedents of selfesteem. Palo Alto, CA: Consulting Psychologists Press.

Gil, F. (1993). Entrenamiento en habilidades sociales. En M. A. Vallejo y M. Ruiz (Eds.). Manual práctico de modificación de conducta. Madrid: Fundación Universidad-Empresa.

Goleman, D. (1998). La práctica de la inteligencia emocional. Barcelona: Kairós.

Maslow, A. (1954). Motivación y personalidad. Madrid, España: Díaz de Santos.

Pelechano, V. (1984). Inteligencia social y habilidades interpersonales. Análisis y Modificación de Conducta, 24(10), 393-420.

Sánchez, H. y Reyes, C. (1986) Metodología y diseño en la investigación científica. Perú: Inide.

Távara Castillo, L. y Torres Oporto, M. del R. (2012). Efectos de un programa de refuerzo de las habilidades sociales en niños considerando la inteligencia emocional (Tesis de maestría). Universidad Femenina del Sagrado Corazón. Lima, Perú.

Ugarriza, N. y Pajares, L. (2006). Adaptación y estandarización del Inventario de Inteligencia Emocional de BarOn ICE: NA, en niños y adolescentes ( $2^{\mathrm{a}}$ ed.). Lima: Libro Amigo.

Vallés, A. (1997). Programa de solución de conflictos interpersonales III. Madrid: EOS

Vallés, A. (2005). Programa de refuerzo de las habilidades sociales I. Madrid: EOS. 